\title{
Exoticism Among the Familiar and Bright
}

\author{
Elizabeth Griffin \\ 6 London Place, Oxford $\mathrm{OX}_{4} \mathrm{1BD}, \mathrm{UK}$
}

\begin{abstract}
The greater the spectroscopic detail observed, the more one appreciates that stars are individuals. Phenomena exhibited by bright stars illustrate some of the wealth yet to be unearthed - and explained.
\end{abstract}

\section{A simple extrapolation}

Many speakers in this conference have used the word 'exotic' as if it means 'bizarre.' Actually, the dictionary definition is 'introduced from abroad; not native.' To an Earthling, therefore, an exotic object is anything from outside the solar system, e.g. stars, and a glance at the brightest ones visible from here indicates that they are all bizarre in some fairly major aspect: Sirius (white-dwarf companion), Arcturus (high-velocity, metal-poor), Vega (Metalpoor; pole-on; CS material), Capella (composite spectrum of two G giants, one rotating rapidly), Rigel (variable RV and line profiles), Procyon (white-dwarf companion), Betelgeuse (variable; spotted), Altair (oblate through rotation), ..., the list goes on. A simple extrapolation leads to the conclusion that the whole lot is weird.

This paper touches on four aspects of observational astrophysics (the local ISM, stellar chromospheres, hot-spot emission, and the evolution of Am stars) which have only recently received quantitative attention. Each contributes significantly to our understanding of the complicated objects we refer to as simple stars, and all of the stars discussed here are brighter than fourth magnitude.

\section{Line asymmetries in Vega and ISM}

By virtue of its brightness, its constancy and its clean, sharp-lined spectrum, Vega has been the standard candle for photometry and a universal comparison for spectroscopy. Unfortunately, Vega is anything but standard; it is metal-poor, probably viewed pole-on, and embedded in débris. It also appears to be situated in a local bubble of $I S$ material.

That the spectrum of Vega shows lines with square profiles was published some years ago (Gulliver, Hill, \& Adelman 1994), and was undoubtedly known for longer by anyone who had observed it at high resolution. The explanation, first suggested by Gray (1988), is that Vega is rapidly rotating but seen poleon; the star is oblate through rotation, so the equatorial region (at the limb) is cooler. The existence of such a temperature gradient produces an enhanced contribution from the stellar limb relative to that from the hotter centre, i.e. a 


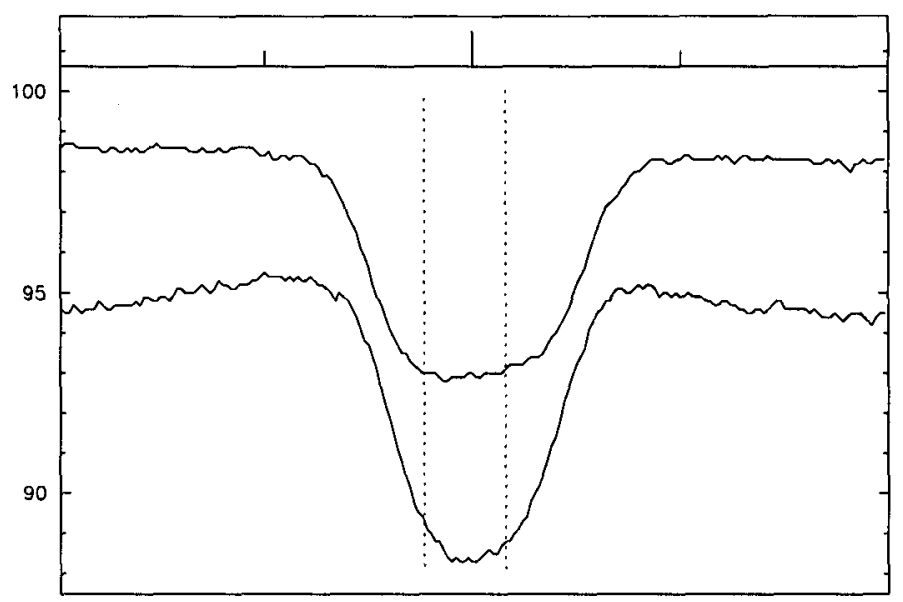

Figure 1. Averaged line profiles in the spectrum of Vega (upper: neutral lines, lower: ionized lines). The dotted lines indicate the extent of the ISM absorption modelled at $\lambda 2344 \AA$ by Lallement et al. (1995).

limb-strengthening. According to Gulliver et al., the effect is only discernible in weak lines, i.e. with central depths of no more than about $3-4 \%$. Throughout the spectrum of Vega one can see faint lines with distinctly trapezoidal profiles.

However, that is not all. Superimposed on those unusual line profiles is an asymmetry as well. It takes the form of an excess depression on the shortwavelength side of the profile, and it seems to be present in lines of all strengths and atomic species. Averaged profiles of neutral and ionized lines (excluding Balmer lines, Ca II and Na I) are reproduced in Fig. 1.

An asymmetry of that nature can be intrinsic to the star: e.g. emanating from a disk swept off the fast-rotating equatorial region. It has been known since the IRAS mission that Vega is embedded in a shell of particulate material. Holland et al. (1998) observed that the shell is a disk containing condensations.

The asymmetry can also be caused extrinsically by $I S$ absorption. Although the distance to Vega is only $7.5 \mathrm{pc}$, there is evidence of $I S$ absorption in the core of Fe II $\lambda 2344 \AA$; Lallement et al. (1995) showed that an observed excess absorption is blue-shifted by a velocity that corresponds closely to that of the local ISM; their best-fitting synthesis suggests three distinct contributions. The dotted lines in Fig. 1 mark the extent of the ISM feature in their model.

\section{Chromospheric eclipses}

A composite-spectrum binary is a system that contains a cool giant primary plus a hot, lower-luminosity secondary. If the orbital inclination is such that eclipses occur, the relatively small secondary is viewed through the extended chromosphere of the primary, giving rise to the highly dramatic phenomenon of chromospheric eclipse. Measurements of chromospheric features provide an exceptional opportunity to study a chromosphere directly. Only 10 such systems 


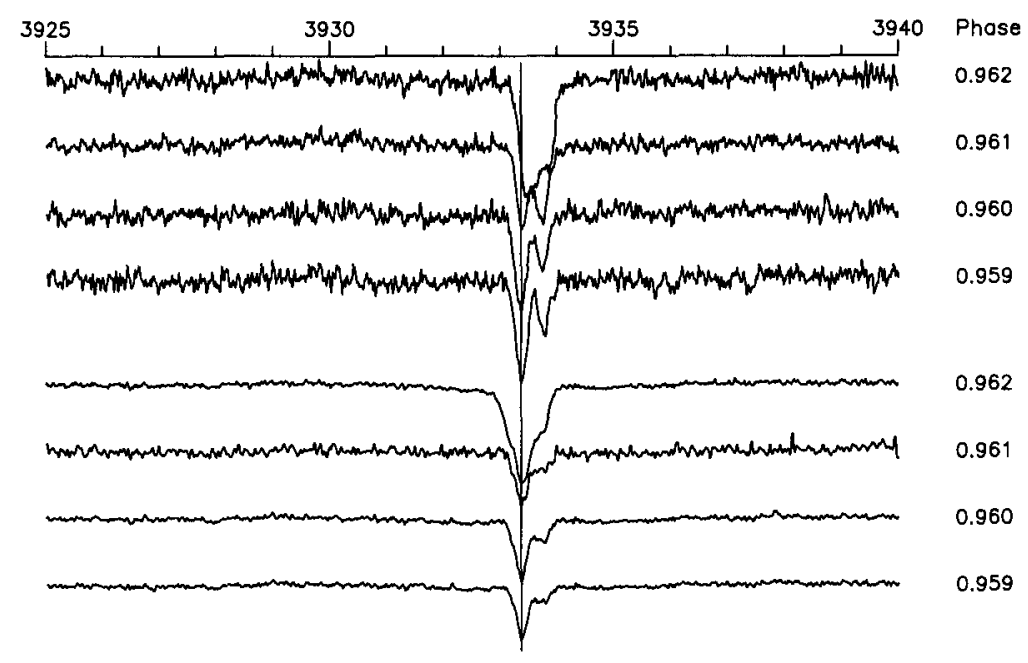

Figure 2. The chromospheric core of the Ca II K-line profile, at the same egress phases in 1990 (upper set) and 1998 (lower set). The feature is seen here against the spectrum of the B6.5 V secondary.

are known that are bright enough for detailed investigation; three ( $\zeta$ Aur, 31 Cyg and $32 \mathrm{Cyg}$ ) are brighter than $m_{V}=4$. While the observed phenomena conform to a generally recognizable pattern, the individual chromospheres manifest a wide range of properties, partly because the primary stars in question cover a range of spectral types and luminosities from late-G giant to M-type supergiant, and partly because a chromosphere has a temperature gradient and a clumpiness that are unique to the underlying star and also vary with time.

Studies of chromospheric-eclipse spectra of $\zeta$ Aur recorded photographically in $1939 / 40,1947 / 8$ and $1955 / 6$ together with more recent observations (1987, $1990,1998,2001)$ indicate a changing chromosphere, both on the time-scale of an orbital period (972 days) and also between ingress and egress (about 38 days). Similar effects are visible in chromospheric spectra of 31 Cyg $(P=10.5$ years) observed in 1972 and 1993 (see Fig. 1, page 388). Studies of the Ca II profile in $\zeta$ Aur also demonstrate changes from night to night, suggesting the presence of small-scale inhomogeneities. Fig. 2 illustrates differences in the chromospheric $\mathrm{K}$-line core of $\zeta$ Aur at identical phases, observed during egress in 1990 and in 1998.

\section{Curious emission in $\mathrm{Si}$}

Under certain conditions in a composite-spectrum binary, a secondary which is hotter than about A0 can stimulate SiI emission in a sub-stellar hot-spot on the giant. Emission at $\lambda 3905.5 \AA$ was first reported in $\zeta$ Aur in 1935 , was rediscovered in 1988 by the author, and has since been monitored frequently and also discovered in three other systems. Its appearance has not been explained quantitatively in the literature, but Harper, Griffin \& Bennett (in preparation) 

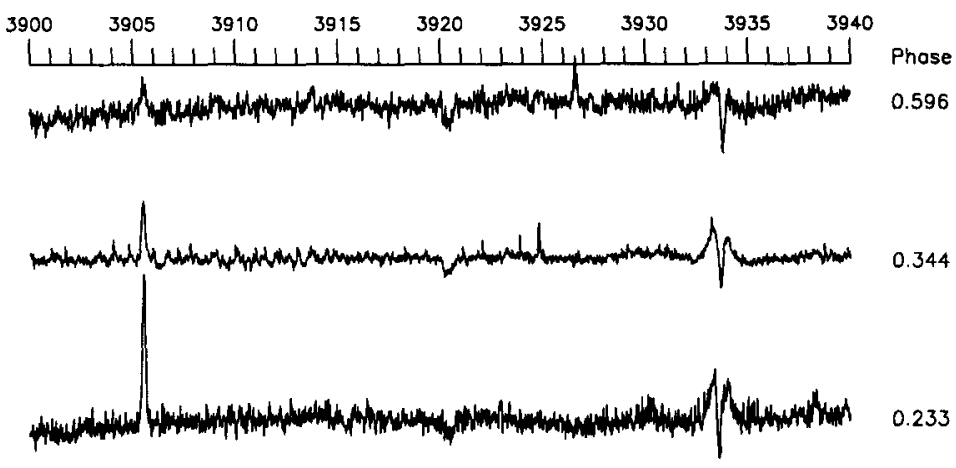

Figure 3. Emission in Si I $\lambda 3905 \AA$ at certain phases in $\zeta$ Aur, shown here against the isolated spectrum of the secondary component. The three spectra have been displaced vertically for clarity.

are able to model the observed emission and its variations in terms of a local over-ionization of $\mathrm{Si}$ I and subsequent cascade. The emission appears to originate in the lower chromosphere of the giant, where ground-state $\mathrm{SiI}$ is plentiful.

Emission occurs in two ground-state lines of Si I: $\lambda 3905.5$ and $\lambda 4102.9 \AA$. $\lambda 4102 \AA$ is very close to the deep core of $\mathrm{H} \delta$ in the secondary's spectrum, but $\lambda 3905 \AA$ occurs in its continuum (see Fig. 3). The geometry of $\zeta$ Aur is particularly favourable for the production of a strong emission that is easily detected in the composite spectrum; in other stars the emission is considerably weaker even at maximum, and may only be detected after the primary spectrum has been subtracted. In any case, the emission at $\lambda 3905 \AA$ is closely blended with a strong photospheric absorption component in the giant, and can only be measured accurately when the primary spectrum has been completely removed.

To an observer, the emission strength is strongly phase-dependent (see Fig. 4), The shape of the dependence depends critically upon the orbital parameters of the binary $-\omega$ and $e$ in particular, and to a lesser extent $i$. Since the emission is very narrow, measurements of its wavelength position offer a method for measuring the rotational velocity, $v \sin i$, of the giant.

\section{An Am giant of near-solar temperature}

There is nothing particularly unusual about a binary consisting of two Am dwarfs, nor about one consisting of Am dwarf + cool giant, but $o$ Leo $\left(m_{V}\right.$ $=3.5$ ) is a case apart. It has been known for a long time as a compositespectrum binary, with RV measurements dating back over 100 years, and its physical properties have recently been determined accurately from a combined astrometric and RV orbit (Hummel et al. 2001); however, the spectral types of the components have remained in question. Its spectroscopy defied separation by the subtraction technique for want of a suitable surrogate primary.

Griffin (2002) reports how the component spectra were eventually separated satisfactorily by the spectrum disentangling technique FDBINARY, a package written by S. Ilijić (University of Zagreb, Croatia) and still under devel- 


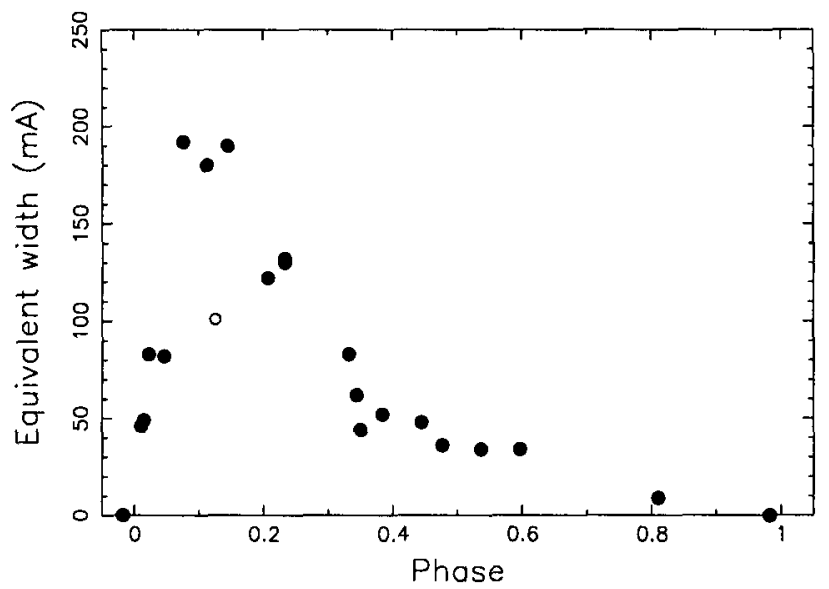

Figure 4. Dependence of the strength of the $\lambda 3905 \AA$ emission with phase. Apart from one highly uncertain point (shown as an open symbol) the scatter is probably real, and reflects variations in the density or temperature of the chromosphere.

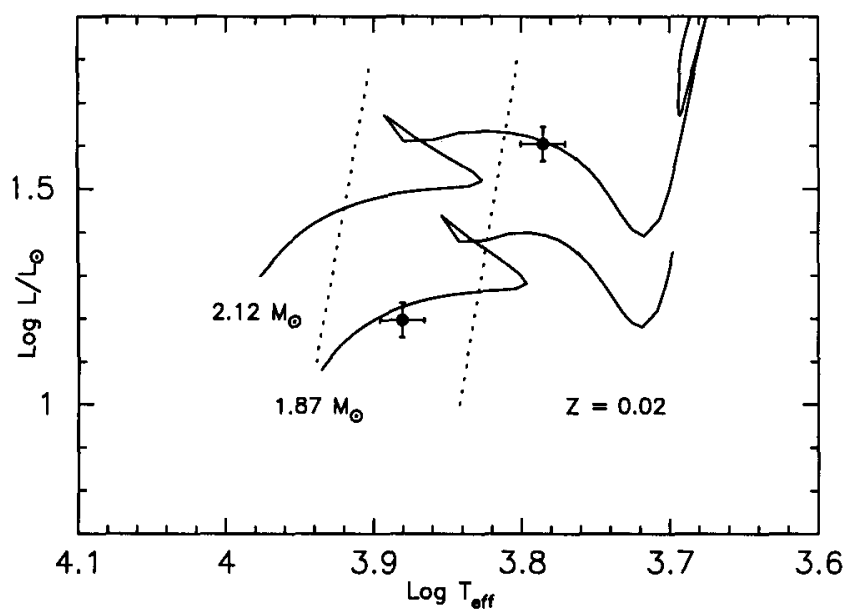

Figure 5. Location of the components of $o$ Leo in the $\log L-T_{\text {eff }}$ plane. The evolutionary tracks, taken from Pols et al. (1998), correspond to the stellar masses derived from the astrometric and RV orbit analysis by Hummel et al. (2001). The dotted lines indicate the boundaries of the instability strip (from Turcotte et al. 2000). 
opment. As Ilijić, Hensberge, \& Pavlovski (2001) explain, FDBINARY identifies the co-moving features by solving simultaneous equations in the Fourier domain instead of wavelength space; it self-consistently extracts the individual components from a series of spectra of the same wavelength region, without requiring a template spectrum. It thus determines the component radial velocities, and recreates the intrinsic spectra, assuming they do not change with time.

The components of $o$ Leo were found to be A7m + F8:m. While the secondary is not unlike many other warm Am stars as regards its physical properties $\left(T_{\text {eff }}=7600 \pm 200 \mathrm{~K}, \log \left(L / \mathrm{L}_{\odot}\right)=1.2, \log g=4.0\right)$, the primary $(o$ Leo $\mathrm{A})$ is highly unusual. With $T_{\text {eff }} \approx 6100 \mathrm{~K}$ and $\log g=3.25$, it should be an early G-type giant, but instead it looks like a very cool Am star; the chemical composition resembles that of an Am dwarf, while the $\mathrm{CH}$ and $\mathrm{CN}$ bands that are becoming prominent in giants of its temperature are not seen. It seems to be in a rapid transitory phase between Am and $\mathrm{G}$ giant: physically like a giant, but chemically still bearing the hall-marks of the Am phase even though it is at, if not below, the limit at which diffusion can operate. Fig. 5 shows the location of the two components of $o$ Leo in the $\log L-\log T$ diagram.

The time-scale of evolution from the instability strip to the lowest point in the red-giant branch is extremely rapid compared to the time taken to reach the instability strip from the main sequence. It seems likely that $o$ Leo A happens to be caught in that transition, and its rarity may partly be due to the rapidity with which such stars pass through that phase. As Keenan \& McNeil (1989) point out, most late- $F$ and early-G giants are rotating rapidly; however, tidal forces may be preventing $o$ Leo A from such rotation. No other Am star is known which shares its properties, so $o$ Leo provides some of the most unusual - and hence valuable - insights we have into the evolution of Am stars.

\section{References}

Gray, R. O. 1988 , JRASC, 82,336

Griffin, R. E. M. 2002, AJ, 123, 988

Gulliver, A. F., Hill, G., \& Adelman, S. J. 1994, ApJ, 429L, 81

Holland, W. S., et al. 1998, Nature, 392, 788

Hummel, C. A., Carquillat, J.-M., Ginestet, N., Griffin, R. F., Boden, A. F., Hajian, A. R., Mozurkewich, D., \& Nordgren, T. E. 2001, AJ, 121, 1623

Ilijić, S., Hensberge, H., \& Pavlovski, K. 2001, in Lecture Notes in Physics, Vol. 573, Astrotomography - Indirect Imaging Methods in Observational Astronomy, ed. H. M. J. Boffin, D. Steeghs, \& J. Cuypers (Heidelberg: Springer), 269

Lallement, R., Ferlet, R., Lagrange, A. M., Lemoine, M., \& Vidal-Madjar, A. 1995, A\&A, 304, 461

Pols, O. R., Schröder, K.-P., Hurley, J. R., Tout, C. A., \& Eggleton, P. P. 1998, MNRAS, 298, 525

Turcotte, S., Richer, J., Michaud, G., \& Christensen-Dalsgaard, J. 2000, A\&A, 360,603 